\title{
Confiabilidade e concordância intra e interavaliadores na avaliação da pressão perineal em nulíparas
}

\author{
Intra- and inter-raters reliability and concordance in the evaluation of \\ perineal pressure in nulliparous
}

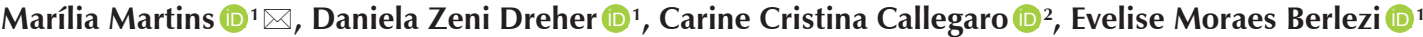 \\ Departamento de Ciências da Vida, Universidade Regional do Noroeste do Estado do Rio Grande do Sul (UNIJUÍ). Ijuí, Rio Grande do Sul, Brasil \\ Departamento de Fisioterapia e Reabilitação, Laboratório de Fisiologia e Reabilitação. Universidade Federal de Santa Maria (UFSM). Santa Maria, \\ Rio Grande do Sul, Brasil
}

Como citar este artigo (How to cite this article):

Martins M, Dreher DZ, Callegaro CC, Berlezi EM. Confiabilidade e concordância intra e interavaliadores na avaliação da pressão perineal em nulíparas (Intra- and inter-raters reliability and concordance in the evaluation of perineal pressure in nulliparous). Sci Med. 2019;29(1):e32614. https://doi.org/10.15448/1980-6108.2019.1.32614

\section{RESUMO}

OBJETIVOS: Verificar a confiabilidade e concordância intra e interavaliadores na avaliação da pressão perineal em nulíparas.

MÉTODOS: Foram incluídas mulheres jovens, saudáveis, nulíparas, não gestantes, que já haviam tido relação sexual e apresentavam correta contração da musculatura perineal ao exame físico. Foram excluídas mulheres que na contração perineal utilizavam outros músculos de forma visualmente perceptível; com alteração do tônus dos músculos pélvicos; com incontinência urinária; com alterações cognitivas; com doença que pudesse afetar os tecidos muscular e nervoso; ou praticantes de atividade física de alto impacto. As participantes foram submetidas a duas avaliações da pressão perineal no mesmo dia, com avaliadores distintos. Após uma semana repetiu-se o protocolo. A pressão perineal foi determinada por meio de um perineômetro e obtida pela diferença entre a pressão máxima (Pmáx) e a pressão mínima (Pmín), registradas pelo aparelho, em milímetros de mercúrio. A contração sustentada (CS) foi avaliada pelo tempo em segundos. Para determinar a confiabilidade foi utilizado o coeficiente de correlação intraclasse (ICC). Para a análise de concordância foi utilizado o teste de Bland-Altman. A comparação de médias foi realizada pelo teste de Wilcoxon. Um valor de $\mathrm{p} \leq 0,05$ foi considerado significativo.

RESULTADOS: Foram incluídas 10 participantes, com média de idade de 23,8 22,9 anos e índice de massa corporal de $22,2 \pm 1,8 \mathrm{~kg} / \mathrm{m}^{2}$. O avaliador A obteve confiabilidade intra-avaliador excelente para Pmín (ICC $=0,86 ; p<0,01$ ) e Pmáx (ICC $=0,92 ; p<0,01)$; muito boa para pressão perineal ( $\mathrm{ICC}=0,65 ; \mathrm{p}=0,01)$; e sem significância estatística para CS. Para o avaliador $\mathrm{B}$, não houve significância estatística para Pmín e Pmáx, mas houve confiabilidade muito boa para pressão perineal (ICC $=0,78 ; \mathrm{p}<0,01)$ e $C S(\mathrm{ICC}=0,70 ; \mathrm{p}<0,01)$. Na análise interavaliadores $(\mathrm{A} v s \mathrm{~B}$ ), no dia 1 não houve significância estatística para Pmín, Pmáx e pressão perineal; mas houve confiabilidade muito boa para CS (ICC=0,71; $\mathrm{p}<0,01)$. No dia 2 não houve significância estatística para Pmín e Pmáx, mas houve confiabilidade muito boa para pressão perineal (ICC=0,62; $\mathrm{p}=0,02)$ e boa para $\mathrm{CS}(\mathrm{ICC}=0,56 ; \mathrm{p}=0,03)$. Houve concordância entre as mensurações intra e interavaliadores.

CONCLUSÕES: A aferição da pressão perineal apresentou confiabilidade intra-avaliador muito boa e interavaliadores boa a muito boa, com concordância intra e interavaliadores.

DESCRITORES: força muscular; distúrbios do assoalho pélvico.

\section{ABSTRACT}

AIMS: To verify intra and inter-rater reliability and concordance in the assessment of perineal pressure in nulliparous women.

METHODS: Young, healthy, nulliparous, non-pregnant women who had had sexual intercourse and had a correct contraction of the perineal musculature on physical examination were included. Women were excluded if they used other muscles in a visually perceptible way during the perineal contraction; with changes in the pelvic muscles tone; with urinary incontinence; with cognitive alterations; with disease that could affect the muscular and nervous tissues; or practitioners of high-impact physical activity. The participants were submitted to two assessments of perineal pressure on the same day, with different evaluators. After one week the protocol was repeated. Perineal pressure was determined by means of a perineometer and obtained by the difference between the maximum pressure (Pmax) and the minimum pressure (Pmin) recorded by the device, in millimeters of mercury. Sustained contraction (SC) was evaluated by the time in seconds. In order to determine reliability, the intraclass correlation coefficient (ICC) was used. Bland-Altman test was used for the concordance analysis. Comparison of means was performed by the Wilcoxon test. A value of $\mathrm{p} \leq 0.05$ was considered significant.

RESULTS: Ten participants were included, with a mean age of $23.8 \pm 2.9$ years and a body mass index of $22.2 \pm 1.8 \mathrm{~kg} / \mathrm{m}^{2}$. The evaluator A obtained excellent intra-rater reliability for $\operatorname{Pmin}(\mathrm{ICC}=0.86, \mathrm{p}<0.01)$ and $\mathrm{Pmax}(\mathrm{ICC}=0.92, \mathrm{p}<0.01)$; very good reliability for perineal pressure $(\mathrm{ICC}=0.65, \mathrm{p}=0.01)$; and no statistical significance for SC. For evaluator B, there was no statistical significance for Pmin and Pmax, but there 
was very good reliability for perineal pressure $(\mathrm{ICC}=0.78, \mathrm{p}<0.01)$ and $\mathrm{SC}(\mathrm{ICC}=0.70, \mathrm{p}<0.01)$. In the inter-rater analysis $(\mathrm{A} v s \mathrm{~B})$, on day 1 there was no statistical significance for Pmin, Pmax and perineal pressure; but there was very good reliability for $\mathrm{SC}(\mathrm{ICC}=0.71, \mathrm{p}<0.01)$. On day 2, there was no statistical significance for Pmin and Pmax, but there was very good reliability for perineal pressure $($ ICC $=0.62, p=0.02)$ and good for $\mathrm{SC}(\mathrm{ICC}=0.56, \mathrm{p}=0.03)$. There was agreement between intra and inter-rater measurements.

CONCLUSIONS: Perineal pressure measurements showed very good intra-rater reliability and good to very good inter-rater reliability, with intra and inter-rater concordance.

KEYWORDS: muscle strength; pelvic floor disorders.

Abreviaturas: CS, contração sustentada; DMD, diferença mínima detectável; DP, desvio padrão; EPM, erro padrão das médias; ICC (intraclass correlation coefficient), coeficiente de correlação intraclasse; Pmáx, pressão máxima; Pmín, pressão mínima; PP, pressão perineal.

\section{INTRODUÇÃO}

A avaliação da musculatura do assoalho pélvico é essencial na prática clínica para identificar disfunções e contribuir para o planejamento, evolução e acompanhamento do tratamento preconizado ao longo do tempo [1]. Qualitativamente, a palpação da musculatura do assoalho pélvico é o padrão adotado para avaliar sua capacidade de contração. Quantitativamente, recursos como dinamômetros, perineômetros e eletromiógrafos podem ser utilizados; no entanto, é a dinamometria que verifica de forma direta a força de contração desses músculos em newtons [2]. Já os perineômetros são capazes de aferir a pressão exercida sobre uma sonda por meio da contração do assoalho pélvico e apresentam importante aplicação clínica no intuito de ensinar e fornecer feedback aos pacientes sobre a efetividade da contração: capacidade de gerar força, sustentar e coordenar estes músculos [3], desde que a musculatura acessória não seja fortemente ativada. Os músculos sinergistas, como o abdômen, adutores, glúteo e músculos respiratórios, são os mais recrutados concomitantemente à contração do assoalho pélvico. Além disso, a presença ou não da manobra de Valsalva pode ser igualmente avaliada por meio da inspeção visual [4]. Os eletromiógrafos também são capazes de fornecer esse feedback, com a vantagem de informar se há ou não contração da musculatura acessória, pois verificam a atividade bioelétrica gerada pelas fibras musculares com eletrodos de superfície que mensuram em microvolts $(\mu \mathrm{V})$ diversas unidades motoras simultaneamente [5]. Há uma boa correlação entre a força muscular e a quantidade de unidades motoras recrutadas [6].
Na prática clínica, a aplicabilidade do dinamômetro não é usual. Esse aparelho é mais utilizado em pesquisas por apresentar bons índices de confiabilidade [2]. Os eletromiógrafos, por sua vez, apesar da sua acurácia, são de alto custo quando comparados aos perineômetros; o aspecto econômico pode ser considerado na escolha por um ou outro equipamento. Nesse sentido, cabe salientar que as técnicas de avaliação devem ser fidedignas, independentemente do equipamento selecionado para complementar a avaliação funcional do assoalho pélvico, uma vez que são parâmetros decisivos na tomada de decisões clínicas.

Assim, a pressão perineal, que é definida como a habilidade de um músculo ou grupo muscular em desenvolver tensão contra a resistência imposta, é resultante de um esforço máximo, tanto dinâmico quanto estático [7]. Pesquisas que avaliem a confiabilidade intra e interavaliadores de cada um desses equipamentos são consideradas importantes [8]. O objetivo deste estudo foi verificar a confiabilidade e a concordância intra e interavaliadores na avaliação da pressão perineal em mulheres nulíparas não gestantes.

\section{MÉTODOS}

\section{Delineamento e ética}

Trata-se de um estudo transversal de confiabilidade e concordância da técnica de aferição da pressão perineal. O estudo foi aprovado pelo Comitê de Ética em Pesquisa da Universidade Regional do Noroeste do Estado do Rio Grande do Sul (UNIJUÍ) sob Parecer Consubstanciado no 1.694.670/2016.

\section{Critérios de elegibilidade}

Foram incluídas mulheres nulíparas, com idade entre 18 a 30 anos, eutróficas, não gestantes, que já haviam tido relação sexual e apresentavam correta contração(grau três ou mais) da musculatura perineal ao exame físico, por meio da Escala de Oxford Modificada [9]. 
Os critérios de exclusão compreenderam mulheres que concomitantemente à contração perineal utilizavam outros músculos, como adutores, glúteos e abdômen, de forma visualmente perceptível. Ainda, foram excluídas mulheres com qualquer tipo de distopia ou alteração do tônus dos músculos do assoalho pélvico, com qualquer tipo de incontinência urinária, com alterações cognitivas que dificultassem a compreensão dos comandos das pesquisadoras, com doença crônica degenerativa que pudesse afetar os tecidos muscular e nervoso, ou que praticassem atividade física de alto impacto, como atletismo, ginástica, jump, kangoo, basquete e vôlei. Respeitando os critérios de elegibilidade, a amostra do estudo foi constituída por 10 mulheres.

O recrutamento da amostra ocorreu por meio da divulgação de folder digital em mídias sociais (aplicativo de mensagem). Esse recurso possibilitou o acesso direto da interessada com a equipe de pesquisa, para ser agendado dia e horário da avaliação, e viabilizou um espaço para dúvidas, resguardado o sigilo e a privacidade. No primeiro contato pessoal, a pesquisadora explicou à participante o protocolo de avaliação. Após, foi formalizada a adesão à pesquisa através da assinatura do Termo de Consentimento Livre e Esclarecido.

\section{Protocolo do estudo}

As avaliações foram realizadas na Clínica de Fisioterapia da UNIJUÍ. As variáveis do estudo foram coletadas a partir da aplicação de um questionário breve, semiestruturado, com questões referentes a dados sociodemográficos, antropométricos, antecedentes ginecológicos, cirúrgicos, de disfunção e desordens da região perineal; e pela avaliação funcional do assoalho pélvico, constituída pela palpação do assoalho pélvico e pela avaliação da pressão perineal.

A avaliação do assoalho pélvico (inspeção visual e palpação) foi realizada por dois avaliadores independentes (cegos entre si) com experiência clínica. As participantes foram submetidas a avaliações de contração voluntária máxima e da capacidade de realizar a contração voluntária sustentada no mesmo dia, por dois avaliadores distintos, denominados como avaliador A e avaliador B, respeitado um intervalo de 10 minutos entre cada avaliador. O protocolo foi repetido após uma semana da primeira avaliação, por ambos os avaliadores.

Para todas as avaliações as participantes foram posicionadas sobre uma maca, em decúbito dorsal com o quadril fletido e abduzido, os joelhos fletidos e os pés em contato com a maca. Os examinadores usaram luvas estéreis para todos os procedimentos.

\section{Avaliação da pressão perineal}

A pressão perineal foi obtida por meio do perineômetro Neurodyn Evolution (Ibramed, Amparo, $\mathrm{SP}$, Brasil). Esse equipamento minimamente invasivo possui uma torneira com três bicos de saída, sendo um conectado a uma seringa de $60 \mathrm{ml}$ e os outros dois a mangueiras, uma ligada ao conector "Pressure", localizado na frente do aparelho, e a outra à sonda de látex vaginal. Para utilizar o equipamento no modo informatizado e realizar a mensuração da pressão perineal, acoplou-se o mesmo ao computador. A pressão perineal é gerada por meio da contração muscular da participante, que produz uma compressão sobre a sonda, mensurada em milímetros de mercúrio (mmHg) [10].

Para mensurar a pressão perineal, a sonda de látex vaginal foi revestida por um preservativo masculino não lubrificado (todos de uma mesma marca), recoberta com gel lubrificante e em seguida colocada lentamente pelo canal vaginal com um movimento de discreta inclinação para baixo até obter seu ajuste à cavidade vaginal. Nesse momento, o software do equipamento já estava em execução na tela do computador, iniciando-se assim a calibragem (mensuração das pressões perineais mínima e máxima). A torneira foi colocada na posição aberta e o êmbolo da seringa foi pressionado vagarosamente até a participante informar sentir a sonda inflada. A percepção da sonda é dependente do tônus muscular, ou seja, cada participante percebeu a pressão da sonda inflada com diferente volume de ar (pressão mínima), o qual foi registrado com vistas a realizar o teste e reteste de mesmo modo em cada participante. Após, a torneira foi fechada e aguardou-se de dois a três minutos para que a sonda entrasse em equilíbrio térmico com o corpo. Uma vez atingido o equilíbrio térmico, clicavase no ícone "Iniciar".

A partir desse momento, foram realizadas cinco mensurações, solicitando-se uma contração rápida, com o máximo de força possível, respeitando um intervalo de 30 segundos de repouso entre as mensurações. Cada participante realizou a contração voluntária máxima com comando verbal para: "apertar e sugar", sendo orientada a realizá-la com o objetivo de comprimir e elevar a sonda no sentido cranial. Na tela do computador foi registrado o pico da pressão perineal em mmHg, gerado em decorrência da contração voluntária máxima que produz uma pressão sobre a sonda [11]. 
A pressão perineal foi estabelecida pela diferença entre os valores da pressão de repouso (registrada no equipamento como "pressão mínima"), que é a gerada pelo avaliador ao inflar a sonda no início do teste, e a pressão máxima que é a gerada pela participante sobre a sonda por meio da contração voluntária máxima. Assim, a pressão perineal foi determinada por meio do cálculo ( $\Delta=$ pressão máxima - pressão mínima) e o melhor resultado, ou seja, o maior valor de pressão perineal entre as cinco mensurações foi utilizado para análise.

Após um intervalo de 90 segundos de repouso, foi avaliada a contração voluntária sustentada (CS) obtida a partir da mensuração do tempo total de contração voluntária da musculatura do assoalho pélvico. Para essa medida foi solicitada a contração com o comando verbal do avaliador de "contrair e sustentar". A participante foi orientada a manter a contração pelo máximo de tempo que conseguisse, com a meta de atingir $50 \%$ da força obtida por ela na última mensuração de contração voluntária máxima. Para esse controle, o avaliador observou ofeedback na tela do computador. Nessa mensuração, permitiu-se uma variação de $10 \%$ para mais ou para menos. O teste foi interrompido quando a participante mostrou-se incapaz de manter os valores de pressão perineal. O tempo de permanência da contração, que foi monitorado pelo avaliador por meio de um cronômetro, foi utilizado como representativo da capacidade de sustentação da contração muscular do assoalho pélvico. Ao término das mensurações, a sonda foi desinsuflada e retirada do canal vaginal.

Os intervalos entre as contrações foram definidos de acordo com a especificidade de cada fibra muscular [12]. Fibras de contração rápida necessitam de um tempo de repouso igual ou duas vezes maior que o tempo em atividade. Essas fibras realizam contrações breves que duram de dois a três segundos. As fibras de contração lenta são resistentes e espera-se que sejam capazes de sustentar uma contração entre oito e 10 segundos; para estas, o tempo de repouso necessário entre as contrações é igual [13]. Por este motivo, visando minimizar o efeito da fadiga muscular nos resultados, optou-se por intervalos ainda maiores, ou seja, 30 segundos entre as cinco contrações de fibra rápida e um descanso de 90 segundos para realizar a contração sustentada.

Para viabilizar a capacidade de sustentação por um período mais prolongado, optou-se por mensurar a contração a $50 \%$ da força máxima. Esta escolha justifica-se pelo fato de que quanto maior a força gerada, maior é o número de unidades motoras recrutadas e maior é o recrutamento de fibras rápidas; ao passo que, em níveis de força mais baixos, maior será o recrutamento de fibras lentas [14], que foi o objeto de análise nessa etapa.

\section{Análise estatística}

Para a construção do banco de dados e análise foi utilizado o programa estatístico SPSS versão 18.0. Para a estatística descritiva utilizaram-se medidas de frequência relativa, medidas de tendência central (média) e dispersão (desvio padrão e intervalo de confiança de $95 \%$ ) observando o tipo de variável. A comparação de médias intra-avaliador e interavaliadores foi realizada pelo teste de Wilcoxon para amostras nãoparamétricas e pareadas com confiabilidade de $95 \%$ considerando que a amostra é pequena.

Para avaliar a confiabilidade e a concordância intra-avaliador foram comparados os resultados das avaliações realizadas pelo avaliador A vs A e pelo avaliador B vs B no primeiro e segundo dia de avaliações, respeitado o intervalo de uma semana, conforme descrito no protocolo do estudo [15]. Para avaliar a confiabilidade e concordância interavaliadores foram comparados os resultados das avaliações do avaliador A $v s$ B no primeiro e no segundo dia de avaliações, respeitado o intervalo de uma semana.

A análise de confiabilidade permite saber o quanto uma medida é consistente e livre de erro em distintas situações. Estudos de confiabilidade permitem definir a utilidade de um equipamento para um ou vários examinadores, possibilitando grande aplicabilidade clínica. A confiabilidade intra-avaliador é aferida quando uma mesma pessoa mede a mesma variável em diferentes situações; a confiabilidade interavaliadores é aferida quando diferentes pessoas obtêm medidas da mesma variável [16]. Para determinar a confiabilidade intra-avaliador e interavaliadores foi utilizado o coeficiente de correlação intraclasse (ICC) com o respectivo intervalo de confiança de $95 \%$. A interpretação dos valores do ICC baseou-se nos critérios estabelecidos por Weir [17] considerando valores de $1,00-0,81=$ excelente; de 0,80-0,61 = muito bom; de 0,60-0,41=bom; de 0,40-0,2=razoável; e de 0,20 a $0,00=$ pobre.

A análise de concordância refere-se à capacidade de aferir resultados idênticos, aplicados ao mesmo sujeito [18]. Para analisar a concordância intra e interavaliadores foi utilizado o erro padrão das diferenças entre duas medidas, ou erro padrão da média (EPM) e a diferença mínima detectável (DMD). O EPM foi calculado dividindo-se o desvio padrão 
(DP) da diferença média pela raiz quadrada de 2 (DP das diferenças $/ \sqrt{2}$ ). A DMD foi calculada utilizando a equação $\mathrm{DMD}=1,96 \times \sqrt{2} \times \mathrm{EPM}$. Ainda, por meio do teste de Bland-Altman, gráficos de dispersão foram construídos e revelaram as diferenças individuais (eixo y) de acordo com as médias observadas nas duas avaliações (eixo $X$ ). A taxa de erro alfa de $p \leq 0,05$ foi considerada significante.

\section{RESULTADOS}

A amostra foi constituída por 10 mulheres com média de idade de 23,8 $\pm 2,9$ (mínima 19, máxima 29) anos e média de índice de massa corporal de $22,2 \pm 1,8 \mathrm{~kg} / \mathrm{m}^{2}$. Nove participantes eram solteiras e oito possuíam ensino superior incompleto. A renda familiar situava-se entre dois e 10 salários mínimos.

A estatística descritiva e as comparações dos resultados obtidos pelo mesmo avaliador entre os dias 1 e 2 estão demonstrados na Tabela 1 . Ao comparar os valores intra-avaliadores observou-se que não houve diferenças significativas tanto no primeiro dia quanto no segundo dia de avaliação.

A Tabela 2 mostra a análise de confiabilidade intra-avaliador. $\mathrm{O}$ avaliador $\mathrm{A}$ obteve confiabilidade excelente na aferição da pressão mínima (Pmín) e da pressão máxima (Pmáx); muito boa na pressão perineal (PP); mas não houve significância estatística para contração sustentada (CS). A confiabilidade do avaliador B não foi significativa na aferição da Pmín e da Pmáx; e foi significativamente muito boa na PP e CS.

A Tabela 3 mostra a análise de confiabilidade interavaliadores. No dia 1 não se obteve significância estatística na análise de confiabilidade relativa às aferições da Pmín, Pmáx; mas obteve-se confiabilidade muito boa e estatisticamente significativa na CS. No dia 2 não houve significância estatística para confiabilidade na Pmín e na Pmáx; mas obteve-se confiabilidade muito boa na aferição da PP e boa na CS. O EPM e a DMD foram baixos na variável PP, tanto no dia 1 como no dia 2.

Tabela 1. Comparação das aferições dos valores de Pressão Mínima, Pressão Máxima, Pressão Perineal e Contração Voluntária Sustentada, na avaliação da pressão perineal em mulheres nulíparas não gestantes, por cada avaliador (A e B) entre os dias 1 e 2.

\begin{tabular}{|c|c|c|c|c|c|}
\hline \multicolumn{6}{|c|}{ Avaliador A } \\
\hline & \multicolumn{2}{|c|}{ Dia 1} & \multicolumn{2}{|c|}{ Dia 2} & \multirow{2}{*}{$\mathbf{p}$} \\
\hline & $\mathrm{M} \pm \mathrm{DP}$ & IC $95 \%$ & $M \pm D P$ & IC $95 \%$ & \\
\hline Pmín (mmHg) & $53,30 \pm 27,47$ & $33,65-72,95$ & $47,70 \pm 23,50$ & $30,89-64,51$ & 0,28 \\
\hline Pmáx (mmHg) & $77,70 \pm 31,97$ & $54,82-100,58$ & $73,50 \pm 28,56$ & $53,07-93,93$ & 0,35 \\
\hline PP (mmHg) & $24,40 \pm 10,01$ & $17,24-31,56$ & $25,80 \pm 10,25$ & $18,47-33,13$ & 0,81 \\
\hline CS (segundos) & $13,50 \pm 21,30$ & $-1,74-28,74$ & $30,90 \pm 51,95$ & $-6,27-68,07$ & 0,21 \\
\hline \multicolumn{6}{|c|}{ Avaliador B } \\
\hline & \multicolumn{2}{|c|}{ Dia 1} & \multicolumn{2}{|c|}{ Dia 2} & \multirow{2}{*}{$\mathbf{p}$} \\
\hline & $M \pm D P$ & IC $95 \%$ & $M \pm D P$ & IC $95 \%$ & \\
\hline Pmín (mmHg) & $57,30 \pm 46,57$ & $23,98-90,62$ & $55,70 \pm 47,61$ & $21,64-89,76$ & 0,87 \\
\hline Pmáx (mmHg) & $81,70 \pm 42,55$ & $51,26-112,14$ & $83,80 \pm 43,97$ & $52,34-115,26$ & 0,95 \\
\hline $\mathbf{P P}(\mathrm{mmHg})$ & $24,60 \pm 11,16$ & $16,61-32,59$ & $28,10 \pm 11,68$ & $19,74-36,46$ & 0,18 \\
\hline CS (segundos) & $24,20 \pm 47,09$ & $-9,49-57,89$ & $40,10 \pm 74,63$ & $-13,29-93,49$ & 0,40 \\
\hline
\end{tabular}

M DDP, média e desvio padrão; IC95\%, intervalo de confiança 95\%; Pmín, pressão mínima; Pmáx, pressão máxima; PP, valor da pressão perineal; CS, contração voluntária sustentada. Teste de Wilcoxon para amostras não paramétricas. Foi considerado como significativo p<0,05.

Tabela 2. Análise de confiabilidade intra-avaliador de cada um dos avaliadores (A e B), na aferição da pressão perineal em mulheres nulíparas não gestantes. O coeficiente de correlação intraclasse refere-se às avaliações realizadas por cada um em dias diferentes.

\begin{tabular}{|c|c|c|c|c|c|c|c|c|c|c|}
\hline & \multicolumn{5}{|c|}{ Avaliador A } & \multicolumn{5}{|c|}{ Avaliador B } \\
\hline & ICC & IC95\% & p & EPM & DMD & ICC & IC95\% & p & EPM & DMD \\
\hline Pmín (mmHg) & 0,86 & $0,54-0,96$ & $<0,01$ & 4,23 & 11,73 & 0,38 & $-0,27-0,80$ & 0,11 & 16,47 & 45,67 \\
\hline Pmáx $(\mathrm{mmHg})$ & 0,92 & $0,71-0,97$ & $<0,01$ & 3,84 & 10,65 & 0,39 & $-0,27-0,80$ & 0,11 & 15,06 & 41,76 \\
\hline $\mathbf{P P}(\mathrm{mmHg})$ & 0,65 & $0,08-0,90$ & 0,01 & 2,40 & 6,65 & 0,78 & $0,33-0,94$ & $<0,01$ & 2,23 & 6,18 \\
\hline CS (segundos) & 0,10 & $-0,53-0,66$ & 0,38 & 16,81 & 46,60 & 0,70 & $0,18-0,91$ & $<0,01$ & 15,11 & 41,90 \\
\hline
\end{tabular}

ICC, coeficiente de correlação intraclasse; IC, intervalo de confiança; EPM, erro padrão da diferença das médias; DMD, diferença mínima detectável; Pmín, pressão mínima; Pmáx, pressão máxima; PP, valor da pressão perineal; CS, contração voluntária sustentada. Foi considerado como significativo p<0,05. 
Tabela 3. Análise de confiabilidade interavaliadores (A vs B) nos dias 1 e 2, na aferição da pressão perineal em mulheres nulíparas não gestantes.

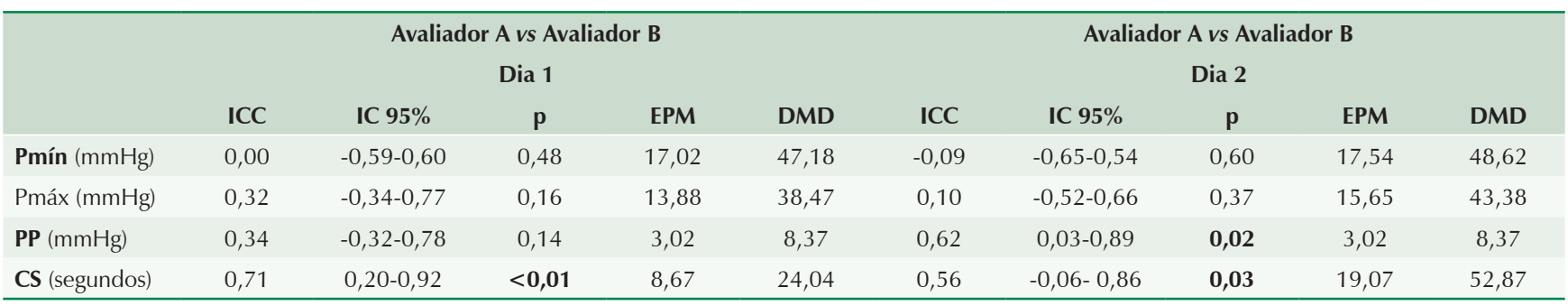

ICC, coeficiente de correlação intraclasse; IC, intervalo de confiança; EPM, erro padrão da diferença das médias; DMD, diferença mínima detectável; Pmín, pressão mínima; Pmáx, pressão máxima; PP, valor da pressão perineal; CS, contração voluntária sustentada. Foi considerado como significativo $\mathrm{p}<0,05$.

A análise de concordância realizada através do método proposto por Bland-Altman [19] está representada nas figuras. A Figura 1 mostra a análise intra-avaliador A da Pmín, Pmáx, PP e CS respectivamente e a Figura 2 mostra a análise intraavaliador B desses mesmos dados. As Figuras 3 e 4 mostram os resultados interavaliadores, nos dias 1 e 2 respectivamente.

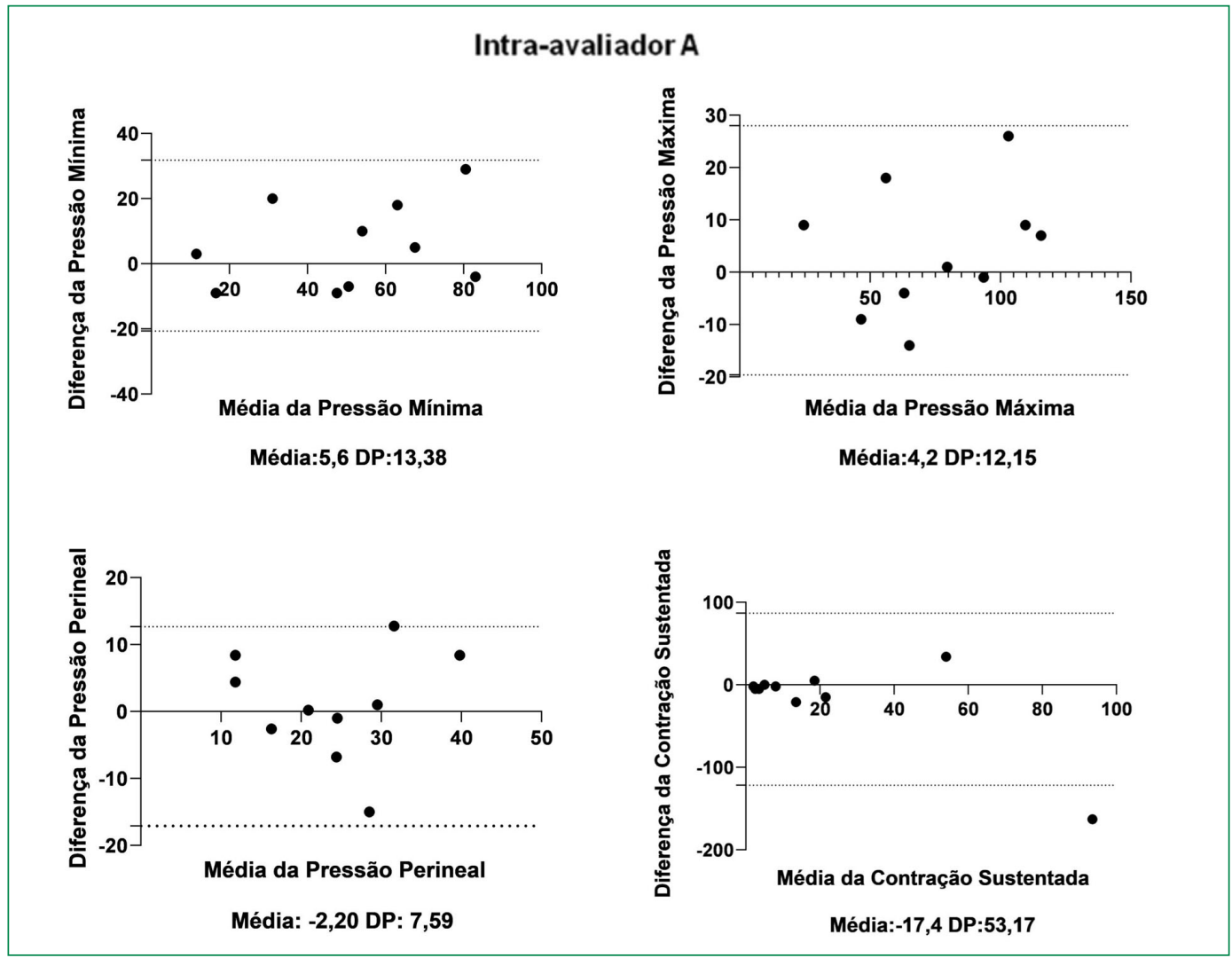

DP, desvio padrão.

Figura 1. Análise de Bland-Altman Intra-avaliador A demonstrando a Pressão Mínima, Pressão Máxima, Pressão Perineal e Contração Sustentada, na medida da pressão perineal em mulheres nulíparas não gestantes. 


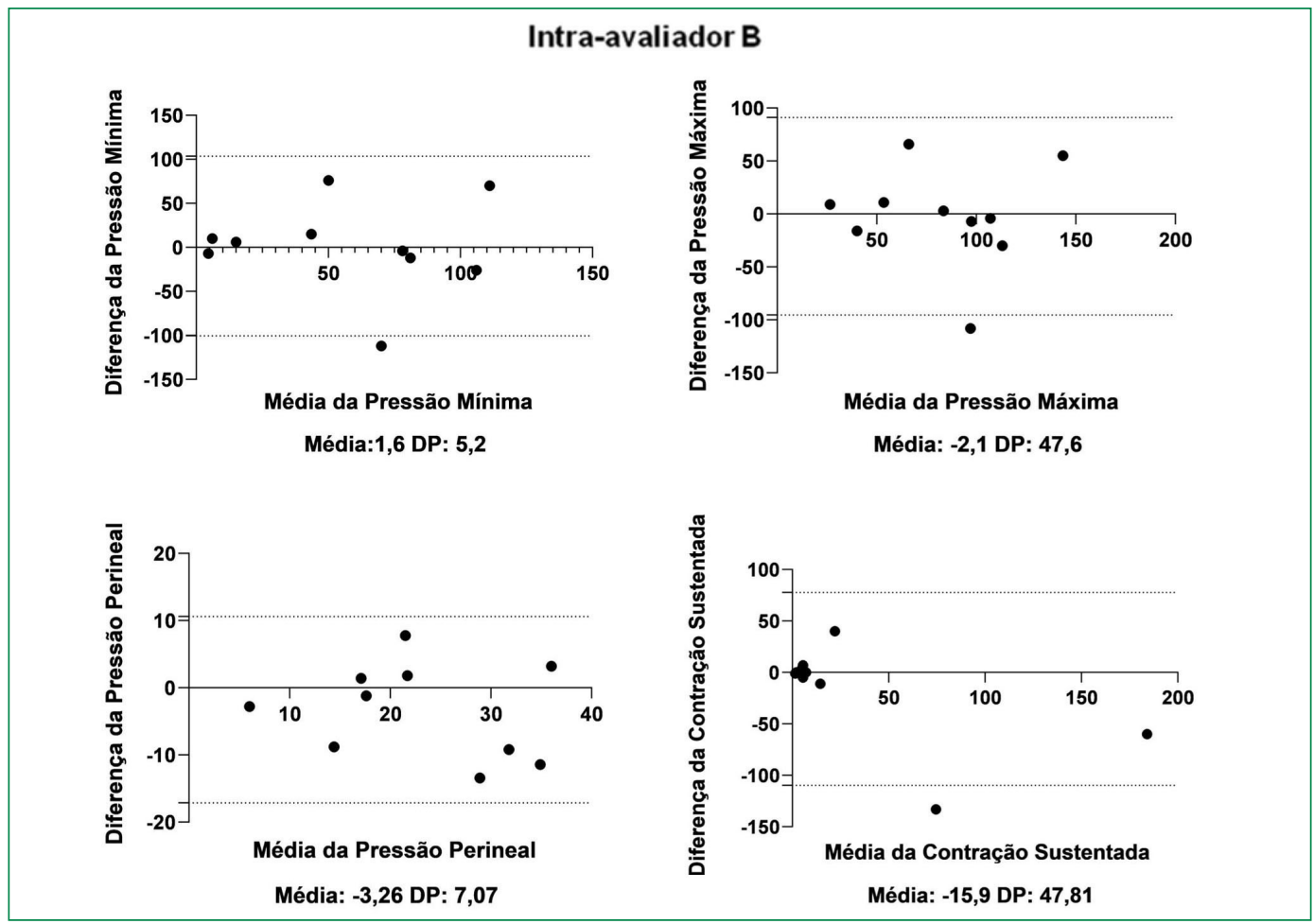

DP, desvio padrão.

Figura 2. Análise de Bland-Altman Intra-avaliador B demonstrando a Pressão Mínima, Pressão Máxima, Pressão Perineal e Contração Sustentada, na medida da pressão perineal em mulheres nulíparas não gestantes.

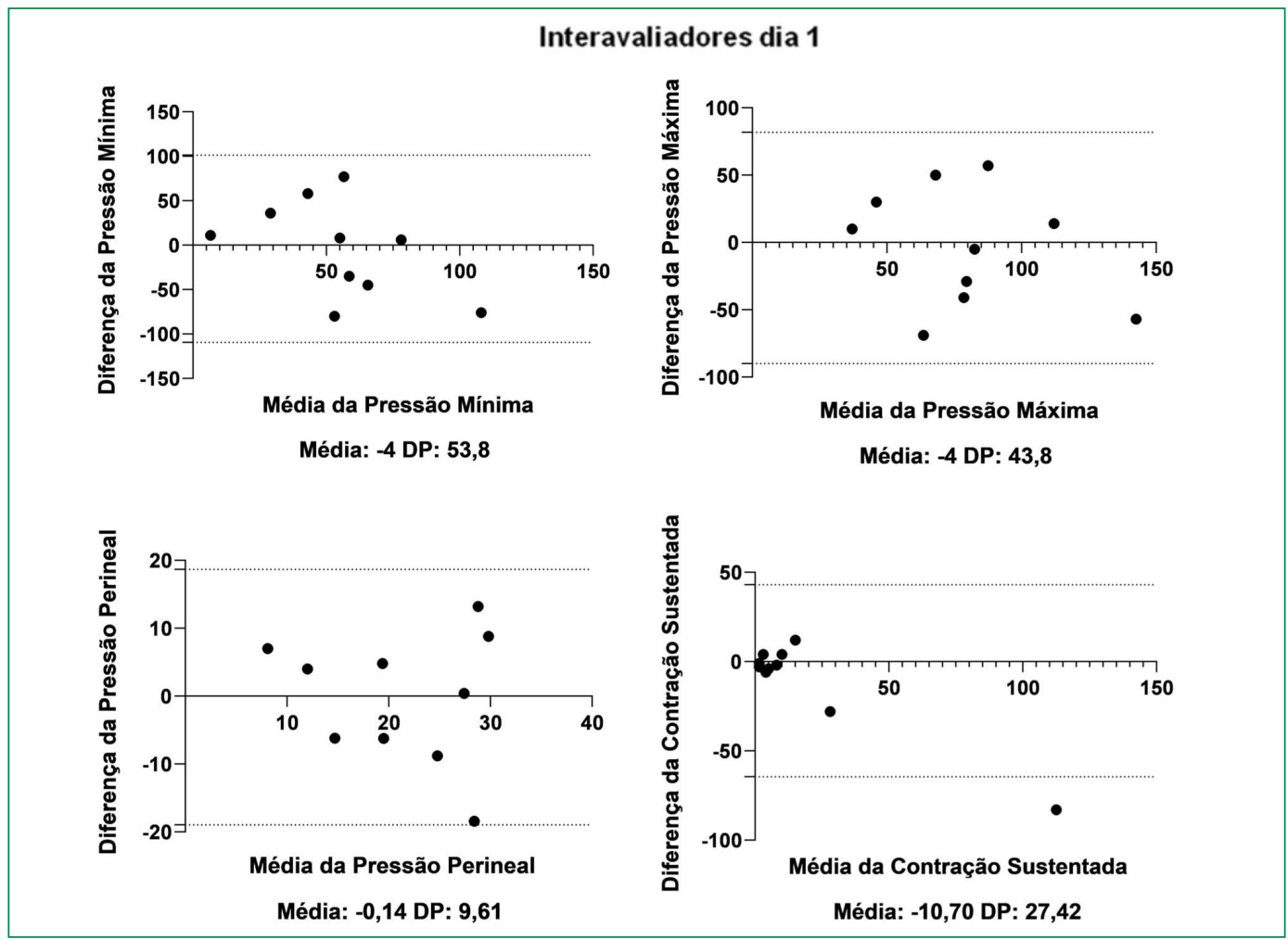

DP, desvio padrão.

Figura 3. Análise de Bland-Altman Interavaliadores A e B no dia 1 demonstrando Pressão Mínima, Pressão Máxima, Pressão Perineal e Contração Sustentada, na medida da pressão perineal em mulheres nulíparas não gestantes. 


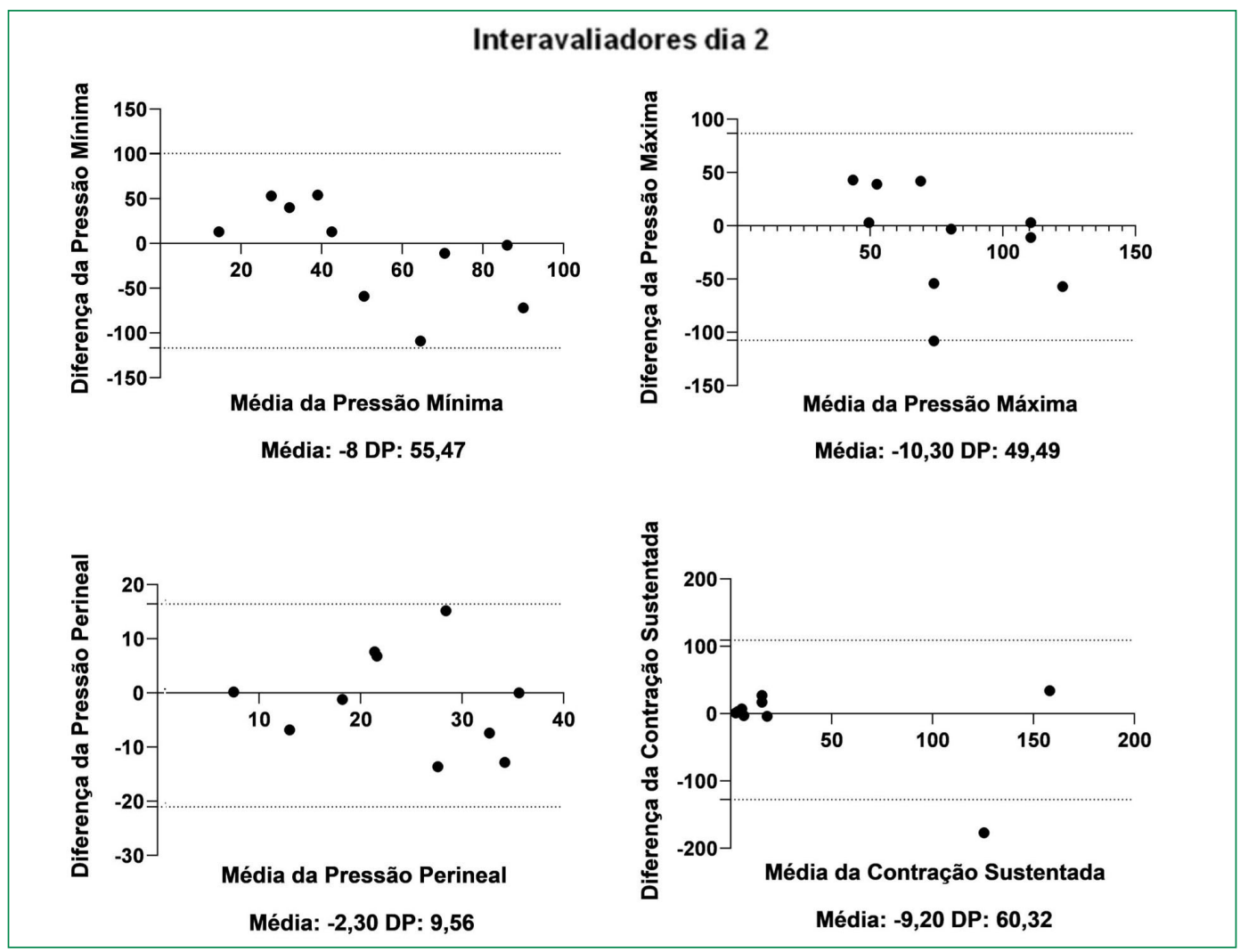

DP, desvio padrão.

Figura 4. Análise de Bland-Altman interavaliadores A e B no dia 2 demonstrando a Pressão Mínima, Pressão Máxima, Pressão Perineal e Contração Sustentada, na medida da pressão perineal em mulheres nulíparas não gestantes.

\section{DISCUSSÃO}

Os resultados revelam que a técnica utilizada neste estudo para a avaliação da pressão perineal intraavaliador mostrou-se confiável, constituindo-se em uma medida capaz de refletir indiretamente a contração voluntária máxima dessa musculatura.

Vários estudos avaliaram perineômetros para mensurar a pressão perineal $[3,8,20-26]$; no entanto, poucos desses pesquisadores utilizaram isoladamente a avaliação da pressão perineal $[3,8,21]$. Um estudo que comparou equipamentos de várias marcas demonstrou que há uma concordância pobre e moderada entre eles no resultado das avaliações [8]. Bo et al. [3] compararam dois perineômetros em seis contrações rápidas realizadas por 20 mulheres com idades entre 21 e 38 anos e concluíram que não foi possível afirmar qual dos dois aparelhos teriam maior validade, uma vez que os mesmos diferem no tamanho da sonda. Outro estudo que avaliou a confiabilidade intra-avaliadores, realizando duas medidas no mesmo dia com intervalo de uma hora entre as mensurações e outra avaliação cinco dias após a primeira, verificou a força e resistência muscular do assoalho pélvico utilizando um perineômetro [21]. As avaliações realizadas no mesmo dia foram muito confiáveis, com coeficiente de correlação intraclasse de 0,95 para a força e 0,94 para a resistência. Entre dias, os valores de correlação permaneceram altos $(0,88$ para força e 0,83 para a resistência), indicando alta confiabilidade [21].

$\mathrm{O}$ valor da PP pode ser um dos parâmetros utilizados para análise na avaliação e comparação dos resultados antes e após as sessões de fisioterapia pélvica. Vale destacar que a confiabilidade intra-avaliadores encontrada para a PP foi muito boa. Apesar de serem observados coeficientes de correlação intraclasse com diferenças expressivas entre os avaliadores A e B para as variáveis Pmín e Pmáx, as mesmas são utilizadas exclusivamente para calcular a pressão perineal [27], não apresentando implicações clínicas.

Contudo, há elementos importantes a destacar, especialmente os que podem apoiar o entendimento da variabilidade observada nas medidas entre os avaliadores. Considerando que ambos os avaliadores 
têm domínio da técnica, uma hipótese para explicar a diferença das aferições pode ser o volume de ar inflado na sonda. O equipamento utilizado não permite o monitoramento do volume de ar: uma vez inflada a sonda e a participante a percebendo cheia, são realizadas as aferições. Deve-se também considerar a possibilidade de existir perda de volume inflado na sonda entre as aferições, salientando-se ainda que o músculo é um tecido distensível, que pode se alongar, e essa capacidade poderia promover adaptações que reduzem a pressão do músculo sobre a sonda; ou seja, de uma mensuração para a outra a pressão pode gerar um resultado diferente, independente do avaliador. Além disso, há variações de ordem física e fisiológica que podem interferir nas aferições, como variações de temperatura corporal e de pressão, superfície e enrijecimento da sonda de látex, associadas às variações não controladas da pressão abdominal [28].

Conforme destacado por Hundley et al. [20], outra preocupação em estudos desta natureza é se o dispositivo em questão está isolando e medindo o parâmetro desejado. Parece que esta questão é de particular importância quando se analisa a contração voluntária máxima do assoalho pélvico, uma vez que este é constituído por uma coleção de vários grupos musculares [20].

Quando analisados os resultados de confiabilidade intraclasse para $\mathrm{CS}$, esta não foi significativa para o avaliador $\mathrm{A}$, o que poderia ser atribuído à participante avaliada, uma vez que o tempo de sustentação depende da capacidade de resistir à fadiga e é individual; ou ao equipamento, e ainda ao poder estatístico da variável. Houve aumento importante da CS da primeira avaliação realizada em cada participante (avaliador A, dia 1) em relação à segunda (avaliador $\mathrm{B}$, dia 1 ), à terceira (avaliador $\mathrm{A}$, dia 2) e à quarta avaliação (avaliador $\mathrm{B}$, dia 2), o que sugere que houve aprendizado da contração voluntária sustentada a partir da segunda avaliação. Assim, a confiabilidade intra-avaliadores no dia 1 ficou comprometida, o que reduziu o poder estatístico dos dados, que foi de apenas $10 \%$, tornando esse dado inconclusivo. Em contrapartida, a confiabilidade intra-avaliadores no dia 2 provavelmente sofreu menor influência da aprendizagem da participante, o que pode ter contribuído para uma confiabilidade muito boa e com poder estatístico de $87 \%$. Já a análise interavaliadores do dia 1 e do dia 2 resultaram em poder estatístico de $99 \%$ e $57 \%$, respectivamente, mostrando que as mensurações da CS podem ser confiáveis quando realizadas pelo mesmo avaliador (avaliador B com poder estatístico suficiente), ou por avaliadores distintos.
Ressalta-se que uma das limitações desta pesquisa está na presença ou não de sinergia durante a contração perineal, variável que neste estudo apenas foi controlada visualmente, constituindo um critério de exclusão. Ainda assim, é importante reconhecer que o aparelho utilizado não permite detectar a presença dos principais músculos que atuam junto com a contração perineal. Por outro lado, dados da literatura mostram que quando isolada a ação desses músculos, restando somente o recrutamento da musculatura perineal com boa eficiência, existe uma forte correlação entre os achados da perineometria e da eletromiografia [29]. Ainda, é importante considerar o constatado por Rahmani et al. [21], que afirmam que o perineômetro pode não ter a precisão desejável, mas isso não impede que as medidas obtidas sirvam de parâmetro na avaliação da pressão perineal.

Compreende-se que o equipamento utilizado neste estudo não permite avaliar e controlar parâmetros que seriam importantes para a precisão e acurácia dos dados obtidos, como monitoramento do volume de ar entre as aferições, considerando que a técnica preconiza que a sonda de látex seja inflada uma única vez, o registro gráfico do trabalho muscular gerado pela pressão sobre a sonda, o tempo de contração sustentada e o recrutamento da musculatura acessória. Além disso, o fato de não ter sido obtida a temperatura corporal das participantes são limitações que podem ter interferido nos resultados do estudo.

O tamanho amostral também poderia influenciar os resultados de confiabilidade obtidos. Contudo, a análise da PP para os avaliadores A e B apresentou poder estatístico de $66 \%$ e $87 \%$, respectivamente. A análise da PP interavaliadores no dia 1 apresentou poder estatístico de $24 \%$ e no dia 2 de $61 \%$. No dia 1 , a análise apresentou baixo poder estatístico, o que pode ter contribuído para a falta de significância estatística. Em contrapartida, o maior poder estatístico dos dados de confiabilidade para análise da PP interavaliadores no dia 2, fortalece os achados de muito boa confiabilidade [30].

Este estudo concluiu que a aferição da pressão perineal apresentou confiabilidade intra-avaliadores muito boa e confiabilidade interavaliadores boa a muito boa, com concordância intra e interavaliadores. Na prática clínica, são necessárias técnicas confiáveis e reprodutíveis para avaliar, prescrever e reavaliar a eficácia do programa de reabilitação, cujas bases tenham evidência científica que as subsidiem. Como a pressão perineal é reconhecidamente importante na prática clínica e é um parâmetro que oferece medidas objetivas, entende-se que a perineometria é uma ótima opção para acompanhar a evolução do tratamento 
fisioterapêutico. Contudo, sugerem-se futuros estudos para que se confirmem estes resultados.

\section{NOTAS}

\section{Apoio financeiro}

Este estudo não recebeu apoio financeiro de fontes externas.

Declaração de conflito de interesses

Os autores declaram não haver conflitos de interesses relevantes ao conteúdo deste estudo.

\section{Contribuições dos autores}

Todos os autores fizeram contribuições substanciais para concepção, ou delineamento, ou aquisição, ou análise ou interpretação de dados; e redação do trabalho ou revisão crítica; e aprovação final da versão para publicação.

\section{Disponibilidade dos dados e responsabilidade pelos resultados}

Todos os autores declaram ter tido total acesso aos dados obtidos e assumem completa responsabilidade pela integridade destes resultados.

\section{REFERÊNCIAS}

1. Menta S, Schirmer J. Relação entre a pressão muscular perineal no puerpério e o tipo de parto. Rev Bras Ginecol Obstet. 2006;28(9):523-9. https://doi.org/10.1590/S0100-72032006000900004

2. Bo K, Berghmans B, Morkved S, KampenMV.Evidence-based physical therapy for the pelvic floor - bridging science and clinical practice. 2a ed. Edinburgh: Elsevier Churchill Livingstone; 2015.

3. Bo K, Raastad R, Finckenhagen HB. Does the size of the vaginal probe affect measurement of pelvic floor muscle strength? Acta Obstet Gynecol Scand. 2005;(84):129-33. https://doi.org/10.1111/j.0001-6349.2005.00676.x

4. Figueiredo EM, Velloso FSB, Vieira GF, Vitória RO, Cruz MC. Avaliação e Diagnóstico Fisioterapêuticos de Mulheres com Disfunções do Assoalho Pélvico. In: Baracho, E. Fisioterapia Aplicada à Saúde da Mulher: 6a ed. Rio de Janeiro: Guanabara Koogan Ltda; 2018. Cap. 29, p. 269-89.

5. Vodusek DB. Electromyography. In: Bo K, Berghmans B, Morkved S, Kampen MV. Evidence based physical therapy for the pelvic floor. Bridging science and clinical practice. London: Churchill Livingstone/Elsevier; 2007. p. 53-63.

6. Vodusek DB. The role of electrophysiology in the evaluation of incontinence and prolapse. Curr Opin Obstet Gynecol. 2002;14(5):509-14. https://doi.org/10.1097/00001703-200210000-00012

7. Amaro JL, Gameiro MO, Kawano PR, Padovani CR. Intravaginal electrical stimulation: a randomized, double-blind study on the treatment of mixed urinary incontinence. Acta Obstet Gynecol Scand. 2006;85(5):619-22. https://doi. org/10.1080/00016340500495058

8. Barbosa PB, Franco MM, Souza FO, Antônio FI, Montezuma T, Ferreira CHJ. Comparison between measurements obtained with three different perineometers. Clinics. 2009;64(6):527-33. https://doi.org/10.1590/S1807-59322009000600007

9. Laycock J, Jerwood D. Pelvic Floor Muscle Assessment: The PERFECT Scheme. Physiotherapy. 2001;87(12):631-42. https://doi.org/10.1016/S0031-9406(05)61108-X

10. Ibramed. Manual de Operação - Neurodyn Evolution. Aparelho de Terapia por Eletro Estimulação. 2009.

11. Martins M, Moraes EB, Dreher DZ. O desempenho da escala de Oxford e do biofeedback manométrico perineal na avaliação da incontinência urinária de esforço em mulheres no período do climatério. Sci Med. 2016;26(1):ID22969. https://doi.org/10.15448/1980-6108.2016.1.22969

12. Baracho E, Botelho S, Nagib ABL. Atuação da Fisioterapia no Tratamento Conservador da Incontinência Urinária Feminina. In: Baracho, E. Fisioterapia Aplicada à Saúde da Mulher: 6 ${ }^{a}$ ed. Rio de Janeiro: Guanabara Koogan Ltda; 2018. Cap. 40, p. 369-76.

13. Seleme M, Valentin EK, Bertotto A, Berghmans B, Uchoa S. Biofeedback Perineal. In: BARACHO, E. Fisioterapia Aplicada à Saúde da Mulher: 6aㅡ ed. Rio de Janeiro: Guanabara Koogan Ltda, 2018. Cap. 30, p. 290-300.

14. Kraemer WJ, Fleck SJ, Deschenes MR. Fisiologia do exercício: teoria e prática. 1ª ed. Rio de Janeiro: Guanabara Koogan; 2013.

15. VanMaanen CJ, Zonnenberg AJ, Elvers JW, Oostendorp RA. Intra/interraterreliability of measurements on body posture photographs. Cranio. 1996;14(4):326-31. https://doi.org/10.1080/08869634.1996.11745985

16. Hobart JC, Lamping DL, Thompson AJ. Evaluating neurological outcome measures: the bare essentials. J Neurol Neurosurg Psychiatry. 1996 Feb;60(2):127-30. https://doi.org/10.1136/jnnp.60.2.127

17. Weir JP. Quantifying test-retest reliability using the interclass correlation coefficient and the SEM. J Strength Com Res. 2005;19(1):231-40.

18. Kottner J, Audigé L, Brorson S, Donner A, Gajewski BJ, Hróbjartsson A, Roberts C, Shoukri M, Streiner DL. Guidelines for Reporting Reliability and Agreement Studies (GRRAS) were proposed. J Clin Epidemiol. 2011;64(1):96-106. https:// doi.org/10.1016/j.jclinepi.2010.03.002

19. Bland JM, Altman DG. Statistical methods for assessing agreement between two methods of clinical measurement. Lancet. 1986;1(8476):307-10. https://doi.org/10.1016/S0140-6736(86)90837-8 
20. Hundley AF, Wu JM, Visco AG. A comparison of perineometer to brink score for assessment of pelvic floor muscle strength. Am J Obstet Gynecol. 2005;(192):1583-91. https://doi.org/10.1016/j.ajog.2004.11.015

21. Rahmani N, Mohseni-Bandpei MA. Application of perineometer in the assessment of pelvic floor muscle strength and endurance: A reliability study. J Bodyw Mov Ther. 2011;(15):209-14. https://doi.org/10.1016/j.jbmt.2009.07.007

22. Bo K, Finckenhagen HB. Vaginal palpation of pelvic floor muscle strength: inter-test reproducibility and comparison between palpation and vaginal squeeze pressure. Acta Obstet Gynecol Scand. 2001;(80):883-7. https://doi.org/10.1034/ j.1600-0412.2001.801003.x

23. Dietz HP, Jarvis SK, Vancaillie TG. The Assessment of Levator Muscle Strength: A Validation of Three Ultrasound Techniques. Int Urogynecol J. 2002;(13):156-9. https://doi.org/10.1007/s192-002-8346-5

24. Kerschan-Schindl K, Uher E, Wiesinger G, Kaider A, Ebenbichler G, Nicolakis P, Kollmitzer J, Preisinger E, FialkaMoser V. Reliability of pelvic floor muscle strength measurement in elderly incontinent women. Neurourol Urodyn. 2002;(21):42-7. https://doi.org/10.1002/nau.2099

25. Frawley HC, Galea MP, Phillips BA, Sherburn M, Bo K. Reliability of Pelvic Floor Muscle Strength Assessment Using Different Test Positions and Tools. Neurourol Urodyn. 2006;(25):236-42. https://doi.org/10.1002/nau.20201

26. Ferreira CHJ, Barbosa PB, Souza FO, Antônio FI, Franco MM, Bo K. Inter-rater reliability study of the modified Oxford Grading Scale and the Peritron manometer. Physiother. 2011;(97):132-8.

27. Martinho NM, Marques J, Silva VR, Silva SLA, Carvalho LC, Botelho S. Intra and inter-rater reliability study of pelvic floor muscle dynamometric measurements. Braz J Phys Ther. 2015;19(2):97-104. https://doi.org/10.1590/bjptrbf.2014.0083

28. Dabbadie L, Seleme M. Biofeedback Perineal. In: Baracho E. Fisioterapia aplicada à Obstetrícia, Urologia e aspectos de Mastologia. Rio de Janeiro: Guanabara Koogan; 2007.

29. Macêdo LC, Lemos A, Vasconcelos DA, Katz L, Amorim MMR. Correlation between electromyography and perineometry in evaluating pelvic floor muscle function in nulligravidas: A cross-sectional study. Neurourol Urodyn. 2018;37(5): 1658-66. https://doi.org/10.1002/nau.23402

30. Button KS, Ioannidis JPA, Mokrysz C, Nosek BA, Flint J, Robinson ESJ, Munafò MR. Power failure: why small sample size undermines the reliability of neuroscience. Nat Rev Neurosci. 2013;14(5):365-76. https://doi.org/10.1038/ nrn3475 